\title{
Uso de la ceniza volante de lignitos (c.v.l.) como materia secundaria. Un resumen considerando especialmente la situación en la República Democrática Alemana (RDA)
}

\begin{abstract}
RESUMEN
La presente publicación es un resumen sobre los trabajos ejecutados para el aprovechamiento de las cenizas volantes de lignito (c.v.l.) en la República Democrática Alemania. Se consideran especialmente los resultados encontrados en patentes de invención y se involucran algunas veces trabajos de otros paises con fines de comparación.
\end{abstract}

Los aspectos claves cubren las investigaciones aplicativas para el uso de c.v.l. en la construcción civil, protección del ambiente y transformaciones quimicas para obtener productos útiies.

Se presentan conclusiones sobre perspectivas y se refleja el importante papel de los análisis físico-químicos para superar el empirismo en relación con el uso de c.v.l.
LOTHAR BEYER, Prof. Dr. en Química Universidad Técnica de Leipzig, Departamento de Ciencias, Cátedra de Química. Karl-Liebknecht-Straße 132, Leipzing DDR-7030, República Democrática Alemana.

\section{INTRODUCCION}

Según los datos de la Empresa VEB Kombinat Braunkohlenkraftwerke (1) 17 Millones de toneladas de c.v.l. aparecen anualmente como el principal subproducto en la industria de la RDA. De éstos, 5,6 millones de toneladas (alrededor de $30 \%$ ) han sido utilizados en el año 1982. Tal porcentaje parece relativamente alto comparado con otros países, por ejemplo con los Estados Unidos [S. Diamond (2)]:

\section{ZUSAMMENFASSUNG}

Die vorliegende Veröffentlichung ist eine

Zusammentassung der in der Deutschen Demokratischen Republik durchgeführten Arbeiten zur Anwendung der Braunkohlenfilterasche (BFA). Besonders werden die in Patenten beschriebenen Ergebnisse berücksichtigt, und zum Vergleich werden einige Arbeiten aus anderen Ländern einbezogen.

Die Hauptaspekte beziehen sich auf angewandte Forschungsarbeiten zur Nutzung der BFA im Bauwesen, zum Umweltschutz und zur chemischen Umwandlung für die Herstellung nützlicher Stoffe.

Es werden perspektivische Schlußfolgerungen gezogen, und es wird die bedeutende Rolle der

physikalisch-chemischen Analytik zur Überwindung einer gewissen Empirie im Zusammenhang mit der Nutzung der BFA herausgestellt.

\section{EINFÜHRUNG}

Nach Angaben des VEB Kombinat Braunkohlenkraftwerke (1) sind die jährlich zirka 17 Millionen Tonnen Braunkohlenfilterasche Hauptabfallstoff der DDR-Industrie. Davon wurden im Jahre 1982 5,6 Millionen Tonnen, das sind zirka $30 \%$, verwertet. Dieser Prozentsatz erscheint relativ hoch im Vergleich zu anderen Ländern, zum Beispiel den Vereinigten Staaten von Amerika 
8 millones, de 48 millones de toneladas, aproximadamente $17 \%$ ].

De ahí resulta la necesidad de investigar profundamente en este campo, con objeto de utilizar la ceniza volante de lignitos en mayor grado para su aplicación práctica, aumentando en esta manera el ingreso nacional.

No se producen cenizas volantes de hulla en la RDA.

De los siguientes aspectos hay que tener en cuenta:

- El uso del contenido de energía involucrado en la ceniza volante y en sus fases silíceas.

- El enriquecimiento de productos valiosos y transformaciones de la materia aprovechables.

- La disminución de la polución del ambiente por la ceniza volante.

No hay duda sobre la función importante de un análisis profundo de la estructura y la composición de la c.v.l. para superar el empirismo en este campo. Mundialmente es conocido que las cenizas volantes presentan composiciones muy diferentes de acuerdo a los yacimientos de lignito, al procesamiento de las excavaciones, al proceso de la combustión y a otros.

En la RDA se han efectuado muchos trabajos de investigación y aplicación a cerca de la ceniza volante, lamentablemente varias veces en forma paralela. Este hecho se origina principalmente en la necesidad económica.

El objeto de este trabajo consiste en presentar un resumen general sobre las múltiples posibilidades de aplicación industrial de la c.v.l. considerando especialmente nuevas tendencias.

Además, se pone especial atención a una valoración crítica de la literatura refiriéndose preferentemente a los trabajos realizados en la RDA. Conocemos el alto nivel, en España, de las investigaciones en el campo de cenizas volantes (ver los más recientes trabajos, entre otros: M. P. de Luxán, M. I. Sánchez de Rojas, F. Soria: "Características de cenizas volantes españolas". Materiales de Construcción 38 (1988), 25-38; A. Pardo, M. P. de Luxán "Normalización española sobre cenizas volantes (normas UNE)", Informes de la
(S. Diamond (2): Von 48 Millionen Tonnen wurden 8 Millionen Tonnen verwertet, das sind ungefähr $17 \%$ ).

Von daher rührt die Notwendigkeit einer umfangreicheren Forschung auf dem Gebiet mit dem Ziel, die Braunkohlenfilterasche in stärkerem Maße für eine praktische Anwendung zu nutzen und damit das Nationaleinkommen zu erhöhen.

Es wird keine Steinkohlenflugasche in der DDR produziert.

Folgende Aspekte müssen berücksichtigt werden:

- Die Ausnutzung des Energiegehaltes der Flugasche und seiner silicatischen Phasen.

- Die Anreicherung von Wertstoffen und die Stoffwandlungen zur Produkterzeugung.

- Die Verminderung der Umweltbelastung durch Flugasche.

Die wichtige Funktion einer leistungsfähigen Stoff-und Struktur-analytik der BFA zur

Überwindung der Empirie auf diesem Gebiet steht außer Frage. Es ist weltweit bekannt, da $\beta$ die Flugaschen eine sehr unterschiedliche

Zusammensetzung je nach

Braunkohlenlagerstätten und Abbautechniken sowie nach der Verbrennungsprozeßführung usw. aufweisen.

in der DDR wurden zahlreiche

Forschungsarbeiten und

Anwendungsuntersuchungen zur Flugasche, gelegentlich parallel, durchgeführt. Dieser Umstand resultiert hauptsächlich aus der volkswirtschaftlichen Notwendigkeit.

Das Ziel dieser Arbeit besteht darin, einen Überblick zu den vielseitigen, industriellen Anwendungsmöglichkeiten der BFA zu geben und dabei besonders neue Tendenzen aufzuzeigen.

Außerdem wird speziell eine kritische Wertung der Literatur mit Bezug auf die in der DDR durchgeführten Arbeiten vorgenommen. Uns ist das hohe Niveau der Forschungen auf dem Gebiet der Flugaschen in Spanien (siehe unter anderen die neueren Arbeiten: M. P. de Luxán, M. I. Sánchez de Rojas, F. Soria: "Charakteristika der spanischen Flugaschen", Materiales de Construcción 38 (1988) 25-38. A. Pardo, M. P. de Luxán "Spanische Norme zu Flugaschen (Normen UNE) "Informas de la construcción 39 (1988) 57-64) und in anderen Ländern bekannt. 
Construcción. 39 (1988) 57-64, y en otros países.

\section{APLICACION DE LA CENIZA VOLANTE EN LA CONSTRUCCION CIVIL}

\subsection{Ceniza volante convencional de lignito.}

Sin duda, en el pasado y en la actualidad, el punto clave de la investigación aplicada respecto a c.v.l. se refiere a la construcción, debido a su carácter como producto silícico con propiedades hidráulicas.

Generalmente, las cenizas volantes de hulla son más apropiadas para tal aplicación que las cenizas volantes de lignito $(3,40)$.

Investigaciones respectivas aparecen hace más de 40 años en la RDA, varias veces en forma repetida (4). Mencionamos algunas publicaciones como resumen (5-11). Otros países, por ejemplo la Republica Popular Democrática de Bulgaria (12), España $(14,15)$ y Japón (13) se dedican a la aplicación práctica de las cenizas volantes como material de construcción civil. Además se usan ventajosamente mezclas de las cenizas volantes de lignito y de hulla para tal fin.

Una tendencia principal de la aplicación en la construcción civil consiste en la sustitución del cemento y de otros materiales hidráulicos (ahorro del cemento).

Otra tendencia sumamente interesante para el futuro es el uso de c.v.l. como agregado muy reactivo con el propósito de inhibir la reacción álcali-sílice.

\section{Cemento/hormigón}

Cronológicamente se reconocen dos etapas principales con respecto al problema en la RDA: 1969-1973 (16-25); 1983-1988 (26-38). Sin embargo, los fundamentos básicos ya se comunicaban con mucha anterioridad:

- F. Heideck (39), en el año 1950, mencionó que la composición diferente y variable de las c.v.l. en Sajonia hace problemático su uso como agregado al cemento para garantizar una calidad alta y estable. En el año 1961, S. Reinsdorf (16) informó sobre la desventaja del hormigón fabricado con c.v.l., debido a su poca estabilidad al ser expuesto a temperaturas bajas en el invierno europeo. Es decir, en casi 35 años de la investigación en este campo de la

\section{ANWENDUNG VON FLUGASCHE IM BAUWESEN}

\subsection{Konventionelle braunkohlenfilterasche}

Zweifellos lag in der Vergangenheit und liegt gegenwärtig der Schwerpunkt der applikativen Forschung im Zusammenhang mit BFA auf dem Sektor Bauwesen wegen ihres Charakters als silicatisches Massenprodukt mit hydraulischen Eigenschaften.

Steinkohlenfilteraschen sind generell besser als Braunkohlenfilteraschen für eine solche Anwendung geeignet $(3,40)$. Entsprechende Forschungsarbeiten werden seit mehr als 40 Jahren in der DDR (4), verschiedentlich in wiederholter Form, durchgeführt, und es sei auf einige Übersichtsarbeiten hingewiesen (5-11). Viele andere Länder, zum Beispiel die Volksdemokratische Republik Bulgarien (12), Spanien $(14,15)$ und Japan (13) beschäftigen sich mit der praktischen Anwendung der Flugaschen als Baumaterial. Außerdem werden vorteilhafterweise Gemische von Braun - und Stein kohlenfilteraschen für diesen Verwendungszweck eingesetzt.

Eine Hauptanwendungstendenz im Bauwesen besteht in der Zementsubstitution und der Substitution von anderen Bindemitteln (Zementeinsparung).

Eine andere, wesentliche zukünftige Nutzungstendenz besteht in der Verwendung von BFA als reaktivem Zusatz zur Minimierung der Alkali-Kieselsäure-Reaktion (AKR).

\section{Zement/Beton}

Zeitlich gesehen heben sich in der DDR zwei Hauptetappen bezüglich der Problembearbeitung voneinander ab: 1969-1973 (16-25); 1983-1988 (26-38). Allerdings werden wesentliche Grundlagen schon sehr viel früher mitgeteilt:

- F. Heideck (39) berichtete im Jahre 1950, da $\beta$ die unterschiedliche und variable Zusammensetzung der BFA in Sachsen ihren Einsatz als Zementzunschlagstoff problematisch in Hinblick auf eine garantierte hohe und stabile Qualität macht. S. Reinsdorf (16) wies im Jahre 1961 auf den Nachteil geringer Frostbeständigkeit des mit BFA hergestellten Betons im europäischen Winter hin. Insofern haben 35 Jahre lang in der DDR durchgeführte 
RDA solamente han sido profundizados tales reconocimientos.

- Muy detallada y resumidamente, E. Fungk, R. Ilgener y E. Lang (17), trataron el problema en la publicación titulada "Cenizas volantes de lignito de la RDA como agregados en la industria del cemento", al final de la década de los 60 .

Ya en este trabajo se clasificaba a las cenizas volantes de la RDA según los depósitos característicos del lignito (Borna, Espenhain, Vockerode, Schwarze Pumpe, Lübbenau, Hagenwerder) y de las diferentes composiciones en 3 grupos.

Existe un catálogo con las propiedades características de estos materiales, tales como durabilidad, relación agua-cemento, contracción para su empleo en cemento puzolánico (18-22, 24). Además, un "catálogo de todas las cenizas de la RDA y su aplicación práctica como material de construcción" es publicado en el año 1985 y sirve para tales fines (11). A base de estos trabajos, las investigaciones más recientes realizadas paralelamente en Weimar $(36,37)$, Cottbus (38) y Leipzig proponen una optimización del uso de la c.v.l. dependiendo de los otros componentes, por ejemplo fluidizantes $(36,37)$ o de las especies características de la c.v.l., respectivamente (29). Algunas empresas industriales ya se ocupan $(30,31)$ con la realización práctica a gran escala. Por ejemplo, se han obtenido buenas experiencias en ensayos técnicos utilizando la c.v.l. de Vockerode (29) en lugar del cemento. En la capital regional Erfurt se han sustituido anualmente más de 5.000 toneladas de cemento por 7.000 toneladas de c.v.l. (30). EI hormigón obtenido de esta manera es conforme con las normas técnicas TGL de la RDA. Según cálculos efectuados (1), algunas especies elegidas de la c.v.l. pueden reemplazar $20-30 \%$ del cemento en la producción del hormigón, en la RDA.

El proyecto TGL 190-72 "Ceniza volantecemento-hormigón” (41), del instituto ORGREB, es una importante propuesta reciente en este campo, y no se esperan más conocimientos básicos en ia RDA.

Una situación completamente diferente y en desarrollo existe respecto a las investigaciones con motivo del uso de c.v.l., muy reactivas para contrarrestar la reacción álcali-sílice [AKR $\left.\left.{ }^{(}\right)\right]$.

(*) $A K R=$ Alkali-Kieselsäure-Reaktion = reaction álcali-sílice.
Forschungsarbeiten auf diesem Gebiet solche schon damals gewonnenen Erkenntnisse nur vertieft.

- Sehr im Detail und zusammenfassend behandelten gegen Ende der 60-iger Jahre E. Fungk, R. Ilgner und E. Lang (17) das Problem "Braunkohlenfilteraschen der DDR als Zuschlagstoffe in der Zementindustrie", wobei schon in dieser Arbeit die Flugaschen der DDR entsprechend der charakteristichen Braunkohlenlagerstätten (Borna, Espenhain, Vockerode, Schwarze Pumpe, Lübbenau, Hagenwerder) und den unterschiedlichen Zusammensetzungen in 3 Gruppen eingeteilt werden.

Es gibt eine Festlegung von Anforderungscharakteristika an die Eigenschaften dieser Materialien, solcher wie zum Beispiel Festigkeit, Wasserbedarf und Schwinden, hinsichtlich ihrer Anwendung in Puzzolanzementen (18-22, 24). Solchen Zwecken dient auch der "Aschekatalog aller BFA der DDR und ihre baustoffliche Nutzung" aus dem Jahre 1985 (11). Basierend auf diesen Arbeiten haben die in jüngerer Zeit parallel in Weimar (36, 37), Cottbus (38) und Leipzing durchgeführten Entwickiungen das Ziel, den Einsatz von BFA in Abhängigkeit von anderen Komponenten, zum Beispiel den Verflüssigern $(36,37)$ oder den Aschesorten (29) zu optimieren. Einige Großbetriebe realisieren schon die technische Umsetzung $(30,31)$. So wurden zum Beispiel gute Erfahrungen mit BFA aus Vockerode bei Großversuchen zur Zementsubstitution gemacht (29). In der Bezirkshauptstadt Erfurt wurden jährlich mehr als 5.000 Tonnen Zement durch den Einsatz von 7.000 Tonnen BFA eingespart (30). wobei der so erhaltene Beton allen technischen Normen "TGL" der DDR entspricht. Nach durchgeführten Berechnungen (1) können einige geeignete BFA-Sorten $20-30 \%$ Zement in der Betonproduktion der DDR ersetzen.

Der vom ORGREB-Institut gefertigte TGL-Entwurf 190-72 "Flugasche-ZementBeton" (41) ist der neueste bedeutende Beitrag auf diesem Gebiet in der DDR, und weitere grundlegende Erkenntnisse sind kaum zu erwarten.

Eine völlig andere, entwicklungsträchtige Situation besteht bezüglich der

Forschungsarbeiten in Hinblick auf den Einsatz hochreaktiver BFA zur Unterbindung der AlkaliKieselsäure-Reaktion (AKR). 
W. Wieker (42) confirmó sin ninguna duda: el agregado de 20-30 \% en masa de c.v.l. de la Empresa Termoeléctrica Hagenwerder al cemento (denominado PZ 9/35 A) disminuye el contenido alcalino en la solución de los poros del hormigón. Resulta una eficiente disminución del resquebrajamiento alcalino (Alkalitreiben), proceso que crea problemas económicos.

\section{Granulación/mortero}

Indicamos los antiguos trabajos básicos $(4,39)$ y los que se ocupan con la resistencia de los morteros elaborados con c.v.l. frente a temperaturas alteradas (43), o frente al ataque de sulfatos (44).

El uso de c.v.l. en el mortero debe involucrar una doble función: la sustitución de aglomerantes hidráulicos y la sustitución de áridos. Debido a la última función, y considerando la economía de transporte junto con la protección del ambiente y el almacenaje, la granulación de la c.v.l. parece ser útil. Ya en 1974 se obtuvieron (45) "pellets" sinterizados de c.v.l. como árido para hormigón ligero. R. Baecaetjev (46) informó en el año 1982, en la patente de invención titulada "Ceniza volante en forma almacenable y el procedimiento de su producción", acerca de un aglomerado de c.v.l. de diferentes tipos. Mediante mezcla con otros aglomerantes hidráulicos como, por ejemplo, elaborado en agua, se podía transformar así en una forma granulométrica aplicable en la construcción civil. En la patente de invención de J. Keuscher (47), titulado "Procedimiento para la preparación de un árido a partir de ceniza" se propone el mezclado de c.v.l. y cemento, junto con un agente hidrófobo para obtener "pellets" que absorben poca o ningun agua. Klings (48) indica, en el año 1983, la granulación de materia pulverizada a granel conveniente de c.v.l.: la ceniza volante al salir pasa, en un sistema cerrado, por una tobera anular que le inyecta agua, conservando de esta manera su fluidez por un tiempo prolongado. La granulación de la c.v.l. es preferente con respecto a la producción de mortero prefabricado (49-51), sin embargo, no exclusivamente (52) es el objeto de varias publicaciones de $\mathrm{W}$. Tischer y M. Munse (Leipzig). Por un pretratamiento de la c.v.l. se ha logrado producir gránulos utilizando un plato pelletero o mediante un quebrantador, produciéndose un mortero seco. La ventaja consiste en que no se precisa el agregado de cal o áridos para el tarrajeado, sino solamente agua y cemento. Sin embargo, ya en el año 1984 (53) W. Zinke (53-57) informó sobre un mortero de c.v.l. utilizable en condiciones meteorológicas especialmente frías y húmedas. El proceso consiste en la
W. Wieker (42) konnte zweifelsfrei nachweisen, da $\beta$ durch Zumahlung von 20-30 Masse-\% BFA des Braunkohlenkraftwerkes Hagenwerder zum Zement (als PZ 9/35 A bezeichnet) der Alkaligehalt in der Betonporenflüssigkeit verringert wird. Daraus ergibt sich eine wirksame Verringerung des Alkalitreibens, eines Prozesses, der ökonomische Probleme verursacht.

\section{Granulierung/Mörtel}

Auf die grundlegenden älteren Arbeiten $(4,39)$ sei ebenso hingewiesen wie auf die Arbeiten zur Beständigkeit von BFA-Mörteln gegenüber Temperaturschwankungen (43) oder gegenüber Sulfaten (44).

Der Einsatz von BFA im Zusammenhang mit Mörtel soll eine Doppelfunktion erfüllen: Die Substitution von Bindemitteln und die Substitution von Zuschlagstoffen. Wegen der letztgenannten Funktion und im Zusammenhang mit der Transportökonomie, dem Umweltschutz und der Lagerfähigkeit scheint eine Granulierung der BFA nützlich zu sein. Gesinterte "BFA-Pellets" als Zuschläge für Leichtbeton wurden bereits im Jahre 1974 dargestellt (45).

R. Baecaetjev (46) beschreibt im Jahre 1982 in einem Patent unter dem Titel "Flugasche in lagerfähiger Form und Verfahren zur Herstellung" ein Agglomerat aus BFA verschiedener Typen. Gemischt mit anderen hydraulischen Bindemitteln, wie zum Beispiel Gips, unter Beimengung von Wasser, läßt es sich in eine granulierte Form überführen, die in der Bauindustrie anwendbar ist. Im patent von J. Keuscher (47) unter dem Titel "Verfahren zur Herstellung eines Zuschlagstoffes aus Asche" wird ebenfalls ein Gemisch aus BFA und Zement zu Pellets verarbeitet, welche durch Zusatz eines Hydrophobierungsmittels nur wenig oder gar kein Wasser aufnemen.

Eine Granulierung von staubförmigem Schüttgut aus BFA beschreibt Klings (48) im Jahre (1983): Die auslaufende Asche wird über eine Ringdüse in einem geschlossenen System mit Wasser benetzt mit dem Ziel, sie über eine längere Zeit hinweg fließfähig zu halten. Die Granulierung von BFA besonders im Zusammenhang mit der Herstellung von Fertigmörtel (49-51), jedoch nicht ausschließlich (52), ist Gegenstand einiger Veröffentlichungen von $W$. Tischer und $M$. Munse (Leipzig). Durch eine Vorbehandlung der BFA wurden unter Benutzung eines Pelletiertellers oder eines Brechers Granulate als Trockenmörtel erzeugt. Der Vorteil besteht darin, daß dieser ohne Zusatz von Kalk oder Zuschlagstoffen auskommt und nur mit Wasser 
preparación de una suspensión homogenéa elaborada a partir de agua a $30^{\circ} \mathrm{C}$ y del aglomerante hidráulico de c.v.l., agregando a tal suspensión los áridos y el cemento. Al mismo tiempo hubo resultados parecidos por otros autores (58-61). La c.v.l. de Thierbach presentó los mejores resultados de prueba respecto a las propiedades del mortero.

Concluyendo se puede resumir que no hay datos exactos sobre la estabilidad a largo plazo de los gránulos y del mortero a base de c.v.l. Es por eso que las valoraciones económicas involucren un alto riesgo. Naturalmente, el agregado del material auxiliar para fomentar la granulación es útil. Sin embargo, en realidad no logrará superar la economía del sistema ceniza/agua.

\section{Materiales aislantes y de relleno}

Encontramos una publicación interesante de H. G. Könnecke (62). Por las propiedades básicas de cenizas volantes de lignito, en una mezcla con el aluminio y el agua se genera hidrógeno gaseoso, el cual funciona como agente espumante formando una materia porosa endurecida y produciendo un aislante térmico de gran capacidad. La ventaja adicional consiste en ser un material incombustible y de fácil fabricación. A pesar de que el hidrógeno se difunde ligeramente por los poros del material endurecido, hay que tener cuidado con las relaciones de los componentes para evitar la formación de gas detonante oxhídrico. Una solución para el futuro podría ser desarrollar gases no combustibles en el medio alcalino.

Los hormigones ligeros resistentes al calor, a partir del portland, y mezclando $37-45 \%$ de c.v.l. aluminosilícicas y $8-20 \%$ de material orgánico inflamable con punto de ignición bajo, por ejemplo, espuma de poliester (63), estos son conocidos (63) como aislantes térmicos. Ademas, hay una cita (64) sobre un material aislante para una red de tuberías y cisternas consistente en un 30-90 \% de c.v.l., 0,5-5\% agente dilatante, arena, cemento y cal. B. Zinke $(1987)(65,66)$ informó tambien sobre los morteros de c.v.l. aislantes térmicos. investigadores de la Union Sovietica (67) usan las cenizas volantes como iellenos para cubiertas blandas de techo compatible con asfalto. und Zement für Putz-und Maurerarbeiten angemacht wird. Ungeachtet dessen wurde schon vorher im Jahre 1984 (53) von W. Zinke (53-57) ein Aschemörtel aus BFA angegeben, der besonders bei kühler und feuchter Witterung einsetzbar ist. Der Herstellungsproze $\beta$ besteht in der Darstellung einer homogenen Suspension von aus $30^{\circ} \mathrm{C}$ angewärmtem Wasser und dem Aschebinder, der dann die Zuschlagstoffe und der Zement zugesetzt werden. Zeitgleich wurden ähnliche Ergebnisse durch andere Autoren (58-61) erhalten. Die BFA von Thierbach ergab die besten Prüfergebnisse zu den Eigenschaften der Mörtel.

Zusammenfassend kann gesagt werden, da es keine exakten Werte zur Langzeitbeständigkeit der Granulate und des BFA-Aschemörtels gibt. Aus diesem Grunde sind die ökonomischen Abschätzungen mit einem hohen Risiko behaftet.

Selbstverständlich ist der Zusatz von Granulierungshilfsstoffen nützlich. Sie übertreffen jedoch nicht die Ökonomie des Systems Asche/Wasser.

\section{isolier-und Füllstoffe}

Eine interessante Veröffentlichung stammt von H. G. Könnecke (62): Wegen der basischen Eigensc'aften von Braunkohlentilteraschen wird in einem Gemisch mit Aluminium und Wasser gasförmiger Wasserstoff entwickelt, welcher als Schäumungsmittel wirkt, wobei eine poröse Masse mit hoher Wärmedämmung nach dem Erhärten gebildet wird. Ein :usätzlicher Vorteil besteht in der Nichtentflammbarkeit des Materials und der leichten Bearbeitbarkeit. Obwohl Wasserstoff leicht durch die Poren des erhärteten Materials diffundiert, ist Vorsicht angeraten bezüglich der Mischungsverhältnisse. um die Bildung von explosivem Knallgas zu vermeiden. Eine zukünftige Lösung könnte die Entwicklung nichtbrennbarer Gase in alkalischem Milieu sein.

Hitzebeständige Silicatleichtbetone aus Portlandzement unter Zusatz von 37-45 Masse$\%$ alumosilicatischer BFA und 8-20 Masse-\% organischen Ausbrennstoffen mit niedriger Züdtemperatur, zum Beispiel Polyesterschaum (63), als Wärmedämmstoffe sind bekannt. Weiterhin gibt es eine Literaturstelle (64) über ein Isoliermaterial für Rohrleitungs-und Behälterisolierung mit 30-90\% BFA; 0,5-5\% Treibmittel, dazu Sand, Zement und Kalk. B. Zinke $(65,66)$ berichtete im Jahre 1987 ebenfalls über wärmedämmende Aschemörtel. Sowjetische Forscher (67) wandten Flugaschen als Füllstoffe tür die Herstellung weicher. bitumenverträglicher Dachbeläge an. 
El relleno con la ceniza volante de lignito en la minería es de actual importancia en la RDA desde el punto de vista de la economía, de la seguridad y de la arquitectura de paisaje.

Se trata en primer lugar de reconocer las propiedades hidráulicas y el comportamiento de c.v.l. bajo altas presiones (69) mediante métodos termoanalíticos (68) considerando el aspecto de aplicación como materiales de relleno en la minería.

\section{Construcción de instalaciones de transporte/construcción subterranea/cerámica gruesa}

Mencionamos el uso de la c.v.l. para capas portantes y de sellado en la construcción de carreteras ahorrando material valioso (70-72) para revestimientos en taludes de construcciones hidráulicas (73), para perforaciones de túnel (74) y para instalaciones de soleras del agua como masa de obturación (75). Para la producción de ladrillos (76) se toman $80 \mathrm{Vol}$ \% de ceniza volante y 20 Vol. \% de barros terciarios del distrito Oberlausitz. Actualmente, se examina el uso de c.v.l. para loza (77).

\subsection{Ceniza volante de lignito proveniente del proceso de adición de caliza.}

El proceso técnico de adición de caliza ("Kalksteinadditiv-Verfahren") es una desulfuración seca del gas de humo proveniente de la combustión del lignito por medio de caliza pulverizada y ha sido elaborado específicamente para lignito de la RDA (78). Actualmente, tal proceso para la RDA parece ser el más económico (79). La ceniza volante proveniente de este proceso es rica en cal, sulfato de calcio y en sulfito de calcio, respectivamente, debido al agregado de unas 0,3 toneladas de cal pulverizada por tonelada de lignito. Esta ceniza volante sirve generalmente como sustituyende del cemento en la construcción civil (80-82).

Hay experimentos para separar las cenizas volantes por medio de una máquina de apagado de cal en dos componentes: uno apropiado para adsorber materia nociva y otro no (83). Así hay intentos de utilizar el producto de desecho. Gran atención se pone al aprovechamiento de la basicidad alta de esta ceniza volante, por ejemplo se propone un proceso para la neutralización de ácido sulfúrico contaminado por sustancias orgánicas (84) o para la purificación de humos ácidos, especialmente de dióxido de azufre
Der Versatz von Flugasche im Bergbau ist in der DDR von aktueller Bedeutung aus ökonomischer Sicht und wegen sicherheitstechnischer und landschaftsgestaltender Relevanz.

Es geht dadei in erster Linie um die Bestimmung der hydraulischen Eigenschaften der BFA in ihrer Funktion als Versatzstoffe und ihr Verhalten unter hohen Drücken (69) mittels thermoanalytischer Methoden (68).

\section{Verkehrsbau/Tiefbau/Grobkeramik}

Erwähnenswert ist der Einsatz von BFA für Trag-Deckschichten im Straßenbau zum Zwecke der Einsparung wertvollen Materials (70-72), für Deckwerke auf Böschungen wasserbaulicher Anlagen (73), im Tunnelbau (74) und für die Herstellung von

Gewässergrund-abdichtungen (75). Für die Ziegelproduktion (76) werden 80 Vol.-\% Flugaschen und 20 Vol.-\% tertiärer Begleitton aus dem Oberlausitzer Revier aufbereitet. Gegenwärting wird die Verwendung von BFA für die Steinzeugproduktion geprüft (77).

\subsection{Kalksteinadditiv-Asche}

Das Kalksteinadditiv-Verfahren zur trockenen Entschwefelung von Rauchgasen aus der Verbrennung der Rohbraunkohle mittels pulverisiertem Kalksteinzusatz wurde speziell für Braunkohlen aus der DDR ausgearbeitet (78). Gegenwärtig sheint dieser Proze $\beta$ für die DDR am ökonomischsten zu sein (79). Die bei diesem Verfahren anfallende Flugasche ist kalkreich und reich an Calciumsulfat und Calciumsulfit wegen des Zusatzes von zirka 0,3 tonnen pulverisiertem Kalk pro Tonne Rohbraunkohle. Diese Flugasche eignet sich grundsätzlich als Zementsubstitut im Bauwesen (80-82).

Es gibt Versuche, um die Flugaschen in zwei Komponenten aufzutrennen unter Anwendung einer Kalklöschmaschine: in eine Komponente, die geeignet ist zur Schadstoffadsorption und in eine andere, die dafür nicht geeignet ist (83). So gibt es Vorhaben, dieses Abfallprodukt $z u$ verwenden.

Große Aufmerksamkeit verdient die Ausnutzung der hohen Basizität dieser Flugasche. Beispielsweise wird ein Proze $\beta$ für die Neutralisation organisch verunreinigter 
$\mathrm{SO}_{2}$. Gana interés en este conexo el aprovechamiento cuádruple de cal en el marco del proceso "de adición de cal" respecto a la desulfurización según:

a) durante la combustión;

b) como cal apagada (83);

c) para prepurificación de los gases de humo (98);

d) como polvo básico en aire (97).

El porcentaje alto de cal en esta ceniza volante abre además otros campos de aplicación práctica.

\section{ENRIQUECIMIENTO DE MATERIAL VALIOSO Y TRANSFORMACION DE CENIZA VOLANTE DE LIGNITO}

\subsection{Fraccionamiento de ceniza volante según principios físicos}

La preparación por separación magnética de la c.v.l. seca, aprovechando el ferromagnetismo de las partículas conteniendo hematita, se ha realizado tecnológicamente produciendo un concentrado de alto porcentaje de hierro $(50-70 \%$ en masa $\mathrm{Fe})$ para la metalurgia (1). Mediante un posterior procedimiento, en un separador por gravedad, se puede fraccionar el resto en dos componentes: una fracción ligera y otra pesada. La fracción ligera puede reemplazar materiales hidráulicos, mientras que la fracción pesada sirve para la aplicación en la industria vidriera, en la cerámica gruesa o, como árido, para hormigón de gas (86).

Existe otra propuesta poco viable, respecto a la separación de la ceniza volante, basada en la desviación de las partículas mediante un campo eléctrico en condiciones de movimiento laminar dentro de un sistema tubular. El principio se basa, tal vez, en el diferente contenido de sulfato de las partículas, lo cual produciría la separación entre cátodo y ánodo en el campo eléctrico (87).

\subsection{Extracción de componentes metálicos}

Un procedimiento para obtener el aluminio o concentrados alumínicos, a partir de barro nativo, mediante extracción en fase líquido-liquido usando aminas como
Schwefelsäure (84) oder ein Proze $\beta$ für die Reinigung saurer Abgase, besonders schwefeldioxidhaltiger $\left(\mathrm{SO}_{2}\right)$, vorgeschlagen. Interessant ist in diesem Zusammenhang die vierfach mögliche Ausnutzung des Kalks bezüglich der Entschwefelung im Rahmen des Kalkstein-Additiv-Verfahrens:

a) während des Verbrennungsprozesses;

b) als gelöschter Kalk (83);

c) zur Vorreinigung der Abgase (98);

d) als basische Staubpartikel in der Luft (97).

Der prozentual hohe Kalkgehalt dieser Flugasche eröffnet außerdem andere praktische Anwendungsgebiete.

\section{WERTSTOFFANREICHERUNG UND STOFFWANDLUNG VON BRAUNKOHLENFILTERASCHE}

\subsection{Fraktionierung von Flugasche nach physikalischen Prinzipien}

Im Rahmen der Trockenascheaufbereitung ist eine Abtrennung hämatithaltiger Partikel unter Ausnutzung des Ferromagnetismus technologisch realisierbar. wobei ein eisenhaltiges Konzentrat hohen

Prozentgehaltes (50-70 Masse-"\% Fe) für die Schwarz-metallurgie bereitgestellt wird (1). Durch ein angeschlossenes Verfahren kann man in einer Schwerkrafttrennanlage den Rükstand in zwei Komponenten fraktionieren, in eine leichte und in eine schwere Fraktion. Die Leichtgutfraktion kann hydraulische Bindemittel substituieren, während die Schwergutfraktion zum Einsatz in der Glas-und Grobkeramikindustrie oder als Zuschlagstoff für Gasbeton empfohlen wird (86).

Es existiert ein anderer, wenig gesicherter, Vorschlag zur Auftrennung von Flugaschen, der darauf beruht, da $\beta$ in Heißwasser eingeschlämmte Aschepartikel in vertikalen Röhren in laminarer Strömung durch ein elektrisches Feld ausgelenkt werden, wobei angenommen wird. daß der Trenneffekt im elektrischen Feld zwischen Katode und Anode auf den unterschiedlichen Sulfatgehalt der Partikel zurückzuführen ıst (87).

\subsection{Extraktion metallhasltiger Komponenten}

Ein Verfahren zur Gewinnung von Aluminium oder aluminiumhaltiger Konzentrate aus einheimischen Tonen mittels Flüssig-FlüssigExtraktion mit Aminen als Extraktionsmitteln ist 
extractantes, se ha introducido en la industria hidrometalúrgica de la RDA por S. Ziegenbalg. Con este fin parece ser muy útil mezclar los concentrados de c.v.l. de alto porcentaje de aluminio. Además se pueden utilizar los concentrados alcalinos de la c.v.l., en el sentido del proceso BAYER. Tecnológicamente parecen ser no realizables las ideas publicadas (88) para combinar dentro de un proceso único la obtención de aluminio, de hierro, de materiales hidráulicos y la purificación de gases de humo a partir de c.v.l.

El bajo contenido de litio en las cenizas volantes exige investigaciones básicas con el fin de una separación de este metal para su aplicación en la industria nuclear.

\subsection{Trasformación del material por tratamiento alcalino}

K.-H. Bergk (89-91) logró la sintesis de zeolitas-A con rendimiento de $50 \%$, a partir de ceniza volante proveniente de Hagenwerder como fuente única de $\mathrm{SiO}_{2}$ y $\mathrm{Al}_{2} \mathrm{O}_{3}$ respectivamente, mediante tratamiento con hidróxido de sodio (89) o con lejía de alta alcalinidad como producto de desecho de la síntesis de zeolitas-A. Como subproducto de la reacción se forman zeolitas- $\mathrm{P}$ e hidroxisodalita.

Las zeolitas son aluminosilicatos hidratados con estructura especial de jaula. Tienen una eminente importancia que irá en aumento para la aplicación industrial como catalizadores, tamices moleculares y adsorbentes (92).

La formación de las zeolitas cristalinas, a partir de las soluciones alcalinas de la ceniza volante, es favorecida por el agregado de cristalitos de zeolita como núcleos de cristalización. Así se puede acortar el tiempo de su formación, como también mediante fuerzas mecánicas (91). Las investigaciones mencionadas se desarrollan con el fin de utilizar los productos obtenidos como adsorbentes baratos descartables (89). De esta manera se contribuye a la protección del ambiente. Paralelamente, el grupo investigador de I.Keil $(93,94)$ se ocupó de problemas parecidos en la síntesis de zeolitas a partir de cenizas volantes.

\subsection{La ceniza volante de lignito en el proceso técnico yeso-ácido sulfúrico}

El método denominado "proceso yeso-ácido sulfúrico Wolfen" basado en el conocido "proceso de Müller-Kühne" (95) mezcla la in der hydrometallurgischen Industrie der DDR durch S. Ziegenbalg eingeführt worden. Deshalb erscheint es sinnvoll, die aluminiumreichen BFA-Konzentrate einzubeziehen. Außerdem können die alkalischen Aufschlüsse der BFAKonzentrate im Sinne des BAYER-Verfahrens eingesetzt werden. Technologisch kaum realisierbar scheinen Vorschläge (88) zu sein, ausgehend von BFA innerhalb eines einzigen Verfahrens die Gewinnung von Aluminium, von Eisen, von hydraulischen Bindemitteln und die Reinigung von Rauchgasen miteinander zu kombinieren.

Der geringe Lithiumgehalt in den Flugaschen erfordert Grundlagenforschungsarbeiten mit dem Ziel der Abtrennung dieses Elementes in Hinblick auf seine Anwendung in der Nuklearindustrie.

\subsection{Stoffwandlung durch alkalischen Aufschlu $\beta$}

K-H. Bergk (89-91) gelang es, aus Hagenwerder-Flugasche als alleiniger $\mathrm{SiO}_{2}$ - und $\mathrm{Al}_{2} \mathrm{O}_{3}-$ Quelle durch Reaktion mit Natronlauge (89) oder mit der alkalischen Mutterlauge als Abfallprodukt aus der ZeolithA-Synthese in etwa 50 \%-iger Ausbeute Zeolith-A zu synthetisieren. Als Nebenprodukt der Reaktion treten Zeolith-P und Hydroxylsodalith auf.

Die Zeolithe sind wasserhaltige Alumosilicate mit käfigartiger Gerüststruktur, die eine eminente, im Anwachsen begriffene, Bedeutung für die industrielle Anwendung als Katalysatoren, Molsiebe und Adsorbentien haben (92).

Die Ausbildung der kristallinen Zeolithe aus den alkalischen Lösungen der Flugaschen wird begünstigt durch das Impfen mit eingebrachten Zeolithkristallen als Kristallkeime. Auch läßt sich

Keimbildung-und-wachstum durch Einwirkung mechanischer Kräfte verkürzen (91). Die aufgeführten Arbeiten gehen in Richtung einer Verwendung der Reaktionsprodukte als billige Einwegadsorbentien (89), und sie leisten gleichzeitig einen Beitrag zum Umweltschutz. Parallel dazu beschäftigte sich die Forschungsgruppe um I. Keil $(93,94)$ mit ähnlichen Problemen der Zeolithsynthese aus Flugaschen.

\subsection{Braunkohlenfilterasche im Gips-Schwefelsäure-Verfahren}

Im sogenannten "Gips-Schefelsäure-Verfahren Wolfen", das auf dem bekannten

"Müller-Kühne-Verfahren" (95) beruht, wird 
reducción de sulfato de calcio con carbón a temperaturas superiores a $700^{\circ} \mathrm{C}$. El gas crudo resultante, conteniendo dióxido de azufre, es transformado a ácido sulfúrico por vía tradicional. Se utiliza el óxido de calcio formado simultánea y conjuntamente con las cenizas volantes de lignito obteniendose, de ambos componentes, un portland con propiedades parecidas al cemento reemplazando, de esta manera, al barro nativo. El nuevo sustituto del cemento tiene características casi iguales al portland original siendo mucho más barato (96).

\section{AMBIENTE Y AGRICULTURA}

Se debe manifestar que la ceniza volante de lignito constituye uno de los principales contaminantes del ambiente, en la RDA, debido al gran volumen producido anualmente. Sobre todo, el polvo de la ceniza suspendido en el aire influye de forma negativa y proviene de los gases de humo, del transporte o los depósitos. Respecto al almacenamiento defectuoso de las cenizas volantes, no se debe olvidar tener en cuenta que la lixiviación a los componentes metálicos les puede influir, tal vez, el agua subterránea. La función dañina, como contaminante del aire, es parcialmente compensada en el sentido de que las partículas alcalinas absorben y neutralizan en el aerosol, por lo menos parcialmente, los gases ácidos provenientes de óxidos de azufre y de nitrógeno (97). Además, las partículas funcionan como núcleos de condensación de lluvia. El aumento de la acidéz de la lluvia se considera también como un efecto del uso de filtros más potentes en las chimeneas (97). Desde este punto de vista las partículas alcalinas de la ceniza volante en aire poseen sin duda un aspecto favorable al medio ambiente.

Se han efectuado varias investigaciones para utilizar ventajosamente las cenizas volantes de lignito en la desulfurización de gases de humo:

- Se usa, por ejemplo ceniza volante fría proveniente del proceso de adición de caliza, haciendo que la corriente de gas de humo la atraviese aumentando así el grado de absorción de dióxido de azufre (98).

- Otro proceso se refiere a la absorción del dióxido de azufre contenido en los humos mediante suspensión acuosa de cenizas, en un sistema continuo que implica la recirculación de la solución acuosa a través de cenizas frescas (99).
Calciumsulfat reduktiv oberhald $700^{\circ} \mathrm{C}$ mit Kohlenstoff gespalten. Das gebildete $\mathrm{SO}_{2}$-haltige Rohgas wird zu Schwefelsäure in traditioneller Weise gewandelt. Das ebenfalls gebildete Calciumoxid kann anstelle des bisher angewandten einheimischen Tons zusammen mit Braunkohlenfilteraschen als

Portlandzementadäquat eingesetzt werden. Das neue Zementsubstitut hat im Vergleich zum Originalportlandzement fast gleiche Eigenschaften und ist sehr viel preiswerter (96).

\section{UMWELT UND LANDWIRTSCHAFT}

Es ist festzustellen, da $\beta$ die Braunkohlenfilterasche einerder wichtigsten Umweltschadstoffe in der DDR aufgrund des großen jährlichen Produktionsvolumens ist. Vor allem beeinflußt der Hauptluftchadstoff Staub, herrührend von Rauchgasen, Transportvorgängen oder Deponien, in negativer Weise. Bezüglich einer nicht sachgemäßen Deponie von Flugaschen darf nicht die Auslaugung von metalihaltigen Komponenten außer Betracht gelassen werden, die unter Umständen das Grundwasser beeinflubt.

Die Funktion als Luftschadstoff wird teilweise kompensiert, weil die basischen Staubpartikel im Aerosol die sauren Gase aus Schwefel-und Stickoxiden adsorbieren und neutralisieren (97). Außerdem wirken die Staubpartikel ais Kondensationskeime für Regen. Die Erhöhung der Acidität des Regenwassers wird so auch auf die Anwendung leistungsstärkerer Filter in den Schornsteinen bei der Kohleverbrennung zurückgeführt (97)

So gesehen besitzen basische Staubpartikel der Asche in der Luft zweifellos einen ambientophilen Aspekt.

Im Anschluß daran wurden verschiedene Forschungsarbeiten durchgeführt, um die Braunkohlenfilterasche in vorteilhafter Weise für die Rauchgasentschwefelung auszunutzen:

- Man benutzt zum Beispiel kalte Kalksteinadditiv-Asche, wobei diese durch den Rauchgasstrom passiert und somit der Einbindungsgrand von Schefeldioxid erhöht wird (98).

- Ein anderes Verfahren betrifft die Absorption von Schwefeldioxid aus Rauchgasen in einer wäßrigen Aschesuspension in einem kontinuierichen Proze $\beta$, wobei die wäßrige Suspension ständig mit trischer Asche kontaktiert wird (99). 
- Otros procesos ventajosos parecidos y específicamente modificados para la desulfurización de gas de humo son: aprovechamiento de gránulos de ceniza como adsorbente (100); combinación de ceniza volante proveniente del proceso de adición de caliza con intercambiadores de calor (101); empleo de las fracciones de ceniza volante con alto porcentaje de óxidos metálicos dispuestas en el conducto de humo $(102,103)$; pretratamiento de la ceniza volante con agregados específicos (104). Finalmente, las mencionadas zeolitas sirven para la desulfurización de gas de humo.

Generalmente se debe considerar el aspecto económico. La eficiencia del proceso en relación al volumen de ceniza volante pretratada, o trasladada, resulta baja respecto al resultado deseado. Los cálculos respectivos no son siempre fiables.

Usando la expresión del premio Nobel Guillermo Ostwald "La exigencia del día" consiste en encontrar catalizadores eficientes para la transformación del dióxido de azufre.

El aprovechamiento de c.v.l. com fertilizante en la agricultura se examina, desde hace tiempo, debido al contenido relativamente alto de cal libre y con el fin de mejorar los suelos ácidos (105). Sin embargo, pensamos que una adición frecuente de tales "fertilizantes" endurece la tierra por largo tiempo, no permitiendo un aumento del rendimiento. Resta la interesante doble función de pellets de c.v.l. como depósito y portador de fertilizantes orgánicos y/o fungicidas, y su aplicación directa como fertilizante anteriormente mencionada.

\section{ANALISIS DE CENIZA VOLANTE DE LIGNITO}

El análisis de cenizas volantes tiene enorme importancia para superar cierto empirismo en su aprovechamiento como material de construcción y otros fines. Se trata de un análisis químico y estructural (107) de c.v.l. conjuntamente con el uso de un proceso computarizado potente (106). Solamente por medio del uso de modernos métodos físico-químicos de análisis se puede optimizar el aprovechamiento; por ejemplo: con la espectrometria de emisión con fuentes de plasma de acoplamiento inductivo (108); con espectroscopía de fluorescencia con rayos $X$
- Weitere, ähnliche, vorteilhafte und speziell auf die Rauchgasentschwefelung modifizierte Verfahren sind: Aschegranulate als Adsorbentien (100); Kombination von Kalksteinadditiv-Asche mit Wärmeaustauschern (101); Einsatz von Filteraschefraktionen mit hohem Metalloxidgehalt im Rauchgaskanal (102, 103); Vorbehandlung von Flugaschen mit speziellen Zusatzstoffen (104). Schlie $\beta$ lich sind die erwähnten, aus Filterasche hergestellten, Zeolithe für die Rauchgasentschwefelung geeignet.

Man mu $\beta$ hauptsächlich den ökonomischen Aspekt berücksichtigen. Die Effizienz des Prozesses bezüglich des Wirkungsgrades ist niedrig im Verhältnis zur Menge vorbehandelter oder transportierter Asche, und die entsprechenden Berechnungen sind sincht immer abgesichert.

Die "Forderung des Tages" - um mit einem Ausspruch des Nobelpreisträgers Wilhelm Ostwald zu sprechen-besteht in der Auffindung hochwirksamer Katalysatoren für die Umwandlung von Schwefeldioxid.

Die Anwendung von BFA als Düngemittel in der Landwirtschaft wird seit einiger Zeit studiert, mit dem Ziel, den relativ hohen Anteil an freiem Kalk für die Verbesserung saurer Böden auszunutzen (105).

Nach unserer Meinung wird jedoch eine Mehrfachausbringung solcher "Düngemittel" den Boden auf längere Sicht betonieren und keine Ertragssteigerung bringen. Es bleibt die interessante Doppelfunktion von BFA-Pellets als mögliche Träger von organischen Depotdüngern und/oder Wirkstoffen zusätzlich zu der bereits erwähnten immanenten Düngerwirkung.

\section{ANALYTIK VON BRAUNKOHLENFLUGASCHE}

Die Analytik von Flugaschen besitzt eine enorme Bedeutung zur Überwindung einer gewissen Empirie bei ihrer Anwendung als Baustoff und für andere Zwecke. Dabei geht es um die Stoff-und Strukturanalytik (107) von BFA mit gekoppelter leistungsfähiger Rechentechnik (106). Nur durch die Anwendung moderner physikalisch-chemischer Analysenmethoden lä $\beta$ t sich der Einsatz der BFA optimieren. Dazu dienen zum Beispiel die Emissionsspektrometrie mit Plasmakopplung (ICP) (108); die

Röntgenfluoreszenzspektrookopie (109); die 
(109); espectroscopía infrarroja y absorción atómica.

Estos métodos, y otros, hoy en día son usuales en los laboratorios que se ocupan con la química analítica de la c.v.l.

Además, los metodos termoanalíticos son de gran valor (68), especialmente en relación a problemas de los materiales de la construcción civil (110). El análisis de procesos para el control de procedimiento y la calidad de los productos adquiere especial importancia debido a la necesidad de mantener constantes los requerimientos técnicos de calidad. Al respecto se ha publicado, recientemente, un proceso rápido de control de calidad para la distribución de tamaño de las partículas fundado en la composición de las fases de las cenizas volantes que utiliza el grado de reflexión de las muestras (111).

\section{BIBLIOGRAFIA}

(1) VEB Kombinat Braunkohlenkraftwerke, Berlín 1984

(2) DIAMOND, S.: Cement \& Concr. Res. 14 (1984) 455-462.

(3) RILEM TC-67: 1983.

(4) W. KRONSBEIN: Zement 30 (1941) 503-506.

(5) PETERS, H.: Betonstein-Zeitung 23 (1957) 27-34.

(6) PAPROCKI, A.: Ton-Ind. Zeitung 23 (1957) 453-456.

(7) JAKOBS, J.: VGB Kraftwerkstechnik 58 (1978) 342-353.

(8) BERRY, E. E., MALHOTRA, V. M.: J. Amer. Conc. Inst. 51 (1980) 59-73.

(9) GOPALAN, N. K., HAGUE M. N.: Cement \& Concrete Res. 15 (1985) 694.

(10) JORKIN, H., SCHUBERT, F.: Betonwerk \& Fertigteiltechn. 51 (1985) 115-120.

(11) VEB Tiefbaukombinat Cottbus, Aschekatalog, Cottbus 1985.

(12) BABACHEV, G. KOLEV, K.: Chem. Abstr. 100 (1983) 11742c.

(13) INOUE, N., TSUNEMATSU, S., HARA, N.: CAJ Reviews 1983, 44-46.

(14) ALONSO RAMIREZ, J. L.: Centr. de ens. de mater. de constr., Madrid, Nº 199 (1969) 1-316.

(15) DE LUXAN, M. P., SANCHEZ DE ROJAS, M. I.: Mater. de Constr. 35 (1985) 3-13.

(16) REINSDORF, S.: Silicattechn. 12 (1961) 484-487.

(17) FUNGK, E., ILGNER, R., LANG, E.: Silicattechn. 12 (1961) 282-307.

(18) FUNGK, E., ILGNER, R., LANG, E.: Silicattechn. 21 (1970) 386-388.

(19) LANG, E.: Baustoffindustrie 13 (1970) 197-201.

(20) KRAUSE, M.: Baustoffindustrie 14 (1971) 19-23.

(21) OEHLSCHLÄGER, A.: Baustoffindustrie 15 (1972) 8-12.

(22) ILGNER, R.: 5. Intern. Baustoff-Silicattag., Weimar, 1973, 463-472.

(23) ALTNER, W., KOENIG, P., GRIEGER: Baustoffindustrie 16 (1973) 17-18.

(24) FUNGK, E., GREULING, P., ILGNER, R.: Baustoffindustrie 17 (1974) B, 17-18.

(25) KOENIG, P.: Baustoffindustrie 20 (1977) B, 15-17.

(26) KRALIK, J.: DDR-WP 212726. (22.8.1984).

(27) HAASE, R.: DDR-WP 225127 (24.7.1985). 
(28) POESCHK, A.: DDR-WP 214608 (17.10.1984).

(29) MEHLHOSE, J.: Wiss. Techn. Inform. Bauwesen, Gera 7 (1984) 6-7.

(30) WESCHKE, K.: Wiss. Techn. Inf. Bauwesen, Gera 7 (1984) 6.

(31) DEEG, S., SIELISCH, N.: Wiss. Techn. Inf. Bauwesen, Gera 7 (1984) 4-6.

(32) GERNHARDT, K.: Diplomarbeit, HAB Weimar, 1984.

(33) ILGNER, R.: Baustoffindustrie 29 (1986) 12-14.

(34) NOACK, E., RICKOWSKY, B., FISCHER, H.: Wiss. Techn. Inf. Bauwesen Gera 9 (1986) 8-12.

(35) KISBAN, G.: 9. Ibausil Weimar 1985, 40-44.

(36) MEIER, F.: Dipiomarbeit, HAB Weimar, 1985.

(37) SCHNEIDER, K.: Diplomarbeit, HAB Weimar, 1985.

(38) ZULEGER, H. D.: Diplomarbeit, IH Cottbus, 1985.

(39) HEYDECK, F.: Silicattechnik 1 (1950) 49-51.

(40) RASCH, R.: Chemikerzeitung-Chem. Appar. 84 (1960) 564-567, 595-598.

(41) ORGREB, TGL 190-72/08, 1986.

(42) WIEKER, W.: Wiss. Koll. TH Leipzig, 20.5.87.

(43) WIEZIG, H. J.: Beton 6 (1970) 233-246.

(44) SCHUBERT, F., LUEHR, H. P.: Betonwerk-und Fertigteiltechn. 45 (1979) 177-182.

(45) KUNZE, W.: Betonwerk-und Fertigteiltechn. 40 (1974) 50-55.

(46) BAECETJEV, R.: OS-P 3242992 (24.5.1984).

(47) KEUSCHER, J.: DDR-WP 220295 (27.3.1985).

(48) KLINGS, M., GOETZE, H. P., WIEKER, G.: DDR-WP 216390 (12.12.1984).

(49) TISCHER, W., MUNSE, M.: DDR-WP 223699 (19.6.1985).

(50) MUNSE, M., TISCHER, W., MUNSE, R.: Baustoffindustrie 28 (1985) 102-104.

(51) MUNSE, M., TISCHER, W., MUNSE, R.: Baustoffindustrie 29 (1986) 4-6.

(52) TISCHER, W., MUNSE, M., LANGE, W.: Baustoffindustrie 29 (1986) 35-37.

(53) ZINKE, B.: DDR-WP 225986 (14.8.1985)

(54) ZINKE, B., LIESKE, H., KOSSATZ, K.: DDR-WP 247890 (22.7.1987).

(55) ZINKE, B., KOSSATZ, K., LIESKE, H., HOFFMANN, W., SCHUHR, U., DIETZKE, S., BUHLS, O.: DDR-WP 247869 (22.7.1987)

(56) ZINKE, B., SCHMIDT, G., DROESE, P., HILBERT, D.: DDR-WP 244545 (8.4.1987).

(57) ZINKE, B., ROSLER, J., SCHMIDT, G., NUBE, K., FRIEBEL, J., SCHUHR, U., LEHM, C., SCHUPP, W., VOIGT, B.: DDR-WP $244546(8.4 .1987)$

(58) LEUSCHNER, H., MITSCHKE, U.: Diplomarbeit, IH. Cottbus, 1984.

(59) KOLBMÜLLER, A., SCHLAFFKE, B., FRÖHLICH, D.: DDR-WP 216002 (8.11.1984).

(60) MUNDRA, E.: DDR-WP 213420 (28.11.1984).

(61) GROB, U., MENZEL, K.: DDR-WP 236085 (28.5.1986).

(62) OTTO, R., HECHT, P., KÖNNECKE, H. G.: DDR-WP 220594 (3.4.1985).

(63) ZINKE, B.: DDR-WP 218279 (30.1.1985).

(64) WELKER, D.: DDR-WP 226279 (21.8.1985).

(65) ZINKE, B., NOACK, H., NOACK, R., KOSSATZ, K., MÜLLER, A.: DDR-WP 247662 (15.7.1987).

(66) ZINKE, B., ZABEL, P., LIESKE, H., MÜLLER, W., STANDTKE, J.: DDR-WP 247893 (22.7.1987).

(67) PERKEV, A. N., PERKEVA, O. A.: Polymer Mater. Moskau 3 (1986).

(68) REINELT, J., WIEKER, W.: DDR-WP 237549 (16.7.1986).

(69) LANG, H.: Diss. A, HAB Weimar, 1983.

(70) SCHUPP, K. P.: Diss. A, IH Cottbus, 1985.

(71) NEUMANN, S.: Diss. A, IH Cottbus, 1985.

(72) PASCH, R.: Wiss. Techn. Inform. Bauwesen Gera, 9 (1986) 6-7. 
(73) SCHUSTER, J.: Diss. A, TU Drseden, 1984.

(74) HUBER, H.: Zement und Beton 23 (1978) 162-170.

(75) SCHMIDT, G., ZINKE, B., WEIDLICH, H. G., SCHNEIDER, J., NIEBE, K., ROESLER, J., MANDREK, V., RAAKE, H., JACKSTEIT, S.: DDR-WP 245001 (22.4.1987).

(76) WENDT, F., HAUGE, R., LINCKE, L.: Wiss. Techn. Inform. Bauund Grobkeramik Weimar 2 (1985) 2-3.

(77) HEIBER, H., MÖRTEL, D.: Baustoffindustrie 29 (1986) 53-54.

(78) KLUGE, W.: Energietechnik 26 (1976) 32-36; 28 (1978) 88-89; 31 (1981) 275-278.

(79) SCHUHMANN, U., TROTHE, R.: Wiss. Techn. Beitr. Abproduktenwirtsch. Berlín 1982, 161-176.

(80) WÄCHTLER, H. J.: Institut für Zement Dessau, 1978.

(81) BARTSCH, D., BOCK, G.: DDR-WP 222292 (15.5.1985).

(82) GEBHART, G., HEIN, K.: DDR-WP 213201 (5.9.1984).

(83) ZILINSKI, E.: DDR-WP 237477 (16.7.1986).

(84) HÄNSGEN, G.:DDR-WP 219167 (27.2.1985).

(85) ZINKE, B.: DDR-WP 208303 (2.5.1984).

(86) ZINKE, B., KOSSATZ, K, WEIDLICH, H.G.: DDR-WP 208302 (2.5.1984).

(87) TISCHER, W., MUNSE, R., MUNSE, M.: DDR-WP 218739 (13.2.1985).

(88) MUNSE, M., TISCHER, W., MUNSE, R., BUCHHOLZ, L.: DDR-WP 212729 (22.8.1984).

(89) BERGK, K. H., PORSCH, M., WOLF, F.: Chem. Techn. 37 (1985) 253-256.

(90) BERGK, K. H., PORSCH, M., WOLF, F.: Chem. Techn. 37 (1985) 425-427.

(91) BERGK, K. H., PORSCH, M., WOLF, F.: Chem. Techn. 38 (1986) 388-391.

(92) PUPPE, L., BÜCHNER, W.: Naturwiss. 71 (1984) 192.

(93) KEIL, I., HADAN, M., RITZMANN, A., FISCHER, F., RÖßLER, P.: Hauptjahrestag. Chem. Gesellsch. DDR, Halle, 1986 , PD7.

(94) KEIL, I., WEIß, E., HORN, A., EISENBRUCH, M.: Hauptjahrestagung Chem. Gesellsch. DDR. Halle, 1986, PD1.

(95) MÜLLER, W. J.: Z. Angew. Chem. 38 (1925) 794-795; KÜHNE, H.: Chem. Techn. Ing. 21 (1949) 227-229.

(96) MYOHL, P., HAASE, R.: Silicattechn. 38 (1987) 241-245.

(97) HARDER, H.: Naturwiss. 71 (1984) 147.

(98) STAUDINGER, G.: DDR-WP 211358 (11.7.1984)

(99) SCHROEDER, G.: DDR-WP 213358 (12.9.1984).

(100) TISCHER, W., STAUDE, J., MUNSE. M., MUNSE, R., NAUMANN, G.: DDR-WP 233630 (5.3.1986).

(101) SCHAUE, A., ALBRECHT, W., HOCKUN, F., KLUGE, W.: DDR-WP 220905 (10.4.1985).

(102) ZILINSKI, E., MÖLLER, D.: DDR-WP 229039 (30.10.1985).

(103) ZILINSKI, E., MÖLLER, D.: DDR-WP 223364 (12.6.1985).

(104) KULBE, B.: DDR-WP 213357 (12.9.1984).

(105) RICHTER, J., RÖNNEBECK: DDR-WP 229109 (30.10.1985).

(106) 2. Intern. Sympos. Fly ash, silica fume, slag and natural pozzolans in concrete, Vol. I and II, Madrid (1986).

(107) KEYN, J., SCHREITER, P., SANSONI, G.: Silicattechn. 36 (1985) 341-343.

(108) SANCHEZ DE ROJAS, M. I., DE LUXAN, M. P.: Mater. Constr. 36 (1986) 31-46.

(109) LECHMANN, E.: Z. f. angewandt. Geolog. 30 (1984) 257-261.

(110) SLANICKA, S.: Thermochim. Acta 93 (1985) 601.

(111) LÖSCHKE, K., WERNER, M., KOSSATZ, K., ZINKE, B.: DDR-WP 234723 (9.4.1986). 